\title{
Correlative Light-Electron Microscopy of Neurons and Brains in Liquid
}

\author{
$\underline{\text { Chikara Sato }}^{1}$, Takaaki Kinoshita ${ }^{2}$, Takeshi Uemura ${ }^{3}$, Naoki Kasahata ${ }^{1}$, Mari Sato ${ }^{1}$, Shoko Nishihara ${ }^{4}$
} and Masami Naya ${ }^{1}$

${ }^{1}$ Biomedical Research Institute, National Institute of Industrial Science and Technology (AIST), Tsukuba 305-8566, Japan.

${ }^{2}$ National Research Center for the Control and Prevention of Infectious Diseases (CCPID), Nagasaki University, Nagasaki 852-8523, Japan.

${ }^{3}$ Division of Gene Research, Research Center for Advanced Science and Technology, Shinshu University School of Medicine, Nagano 390-8621, Japan.

${ }^{4}$ Laboratory of Cell Biology, Soka University, Hachioji, Tokyo 192-8577, Japan.

The identification of proteins and its locational changes is necessary to fully understand the behavior and function of cells and tissues. This can be idealistically accomplished by Correlative Light-Electron Microscopy (CLEM). In this method, high resolution nature of Electron Microscopy (EM) was combined with multi color-labeling of Fluorescence Microscopy (FM). Among CLEM, most straightforward method for biological sample is to perform microscopy of samples in liquid because cells are basically live in an aqueous environment. Atmospheric scanning EM (ASEM) was developed for in situ CLEM observation of wet sample immersed in solution. Here, we demonstrate FM to EM, and electron-beam induced labeling and FM of cells and tissues in aqueous solution using ASEM (Fig.1 left).

FM to EM observation was performed for the aldehyde-fixed primary cultured neurons using ASEM ClairScope $^{\mathrm{TM}}$ (JEOL Ltd.). ASEM allows the inverted SEM observes the wet sample from below, while an optical microscope observes it from above quasi-simultaneously. The disposable ASEM dish with a silicon nitride ( $\mathrm{SiN}$ ) film window can be coated variously to realize the primary-culture of substratesensitive invertebrate and mammal neurons in a few milliliters of culture medium in a stable humidified $\mathrm{CO}_{2}$ incubator environment. CLEM observation was performed for aldehyde-fixed neurons in radical scavenger buffer, and for fixed mouse brains in pyrene-reagent solution using ASEM, as follows.

FM to EM: Neuron differentiation including axonal partitioning, was studied using primary-culture neurons, and presynaptic induction by Glutamate Receptor $\delta 2-\mathrm{N}$-terminus domain-linked fluorescent magnetic beads was correlated to the presynaptic-marker Bassoon. To visualize intra-axonal segmentation, primary cultured Drosophila neurons were fluorescence-gold-labelled using anti-BP102 glycan antibodies and observed using both ASEM and its FM. Neurons cultured on a poly-DL-ornithine on the SiN film were labeled by mouse anti-BP102 antibody / anti-mouse Fab' FluoroNanogold (red) and also by rabbit anti-HRP antibody (green) [1]. FM showed that BP102s are basically localized in the proximal region of an axial fiber (Fig. 1B), although HRP epitopes are localized in the whole axial fiber (Fig. 1A). ASEM visualized that BP102s are accumulated in the proximal region of the intra-axonal boundary (Fig. 1C arrow), forming a hexagonal frame (arrowhead). The segmentation is shown to be highly related to the spectraplakin protein [2] which supports the cell trafficking from microtubule route to actin route.

Neurons transfected by Sindbis-EGFP were cultured with fluorescent magnetic beads coated with GluR $\delta 2-N T D-F c$, and immuno-stained against presynaptic vesicular glutamine transporter 1 (VGluT1) and/or presynaptic active zone protein Bassoon [3]. FM showed presynaptic fibers leading to bright

(C) The Author(s), 2021. Published by Cambridge University Press on behalf of Microscopy Society of America 
spherical magnetic beads (Fig. 1D-E). ASEM showed that presynaptic proteins presumably of an indistinguishable neurite branch surrounded each bead when a neurite attached to it (Fig. 1F).

EM to FM: We synthesized a new pyrene-based fluorescent probe targeting SH groups. This labeling employed thiol-ene click reactions between the probes and SH groups and was triggered by radicals generated by UV light or an electron beam. When two tagged pyrene groups on a protein were close and formed an excimer, they showed red-shifted fluorescence; the shift of two SH residues to within $\sim 35 \AA$ during protein movement can be monitored. Radicals formed in liquid by the electron beam, caused the thiol-ene click reactions, and the excimer fluorescence of the labeled proteins in sectioned brains were visualized by FM (Fig. 2) [4]. The area scanned at low magnification by the inverted SEM was imaged brighter than the surroundings, and the area scanned at higher magnification was imaged more brighter in Fig. 2D. Since the fluorescent labeling is induced by a narrow EM beam, high spatial resolution labeling is expected. This method is expected to be employed to label single neuron, organelle and bacterium.

\section{References:}

[1] T Kinoshita et al, Microscopy and Microanalysis 20 (2014), p. 469.

[2] T Kinoshita et al, Scientific Reports 7 (2017), p. 41455.

[3] K Hirano et al, Ultramicroscopy 143 (2014), p. 52.

[4] M Naya, C Sato, Int J Mol Sci 21 (2020), p. 7550.

[5] The authors acknowledge fundings (KAKENHI Grants from JSPS: 19K10091, 19H04209 to CS).



Figure 1. Axonal segmentation by FM (A-B) and ASEM (C) [1]. Distribution of Bassoon and VGluT1

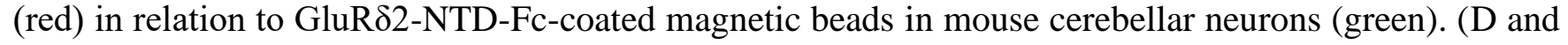
E) FM. (F) Distribution of Bassoon tagged with Alexa 594 and Nanogold, visualized by ASEM. Bassoon (red) was observed as a gathering of white dots presumably in the axonal connection with the beads [3].
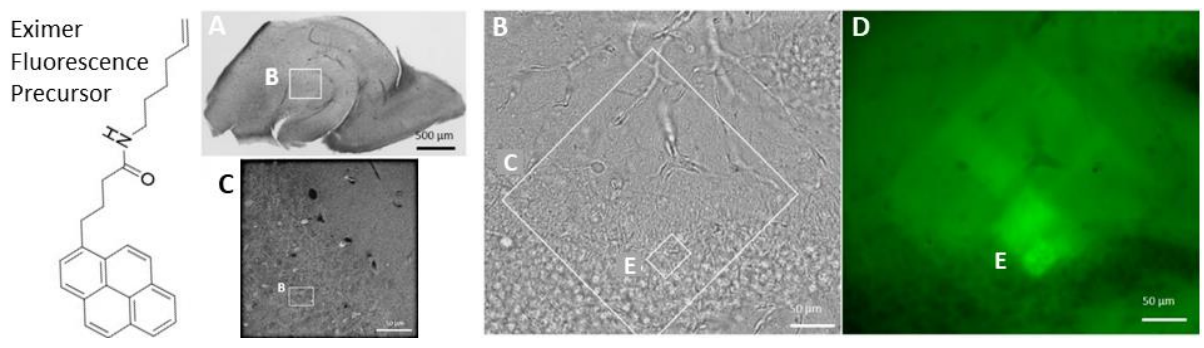

Figure 2. Mouse hippocampal tissue imaged by the electron beam-induced EFP labeling and CLEM. The tissue was GA-fixed, reduced, PCG/PTA stained (A-C) and EFP-labeled using the electron beam of ASEM. (A) Phase contrast OM. (B-C) ASEM images. (D) FM of the tissue after the electron scan [4]. 